\title{
Mechanism Mobility and a Local Dimension Test
}

\author{
Charles W. Wampler ${ }^{1,2, *}$ \\ General Motors Research and Development, Mail Code 480-106-359, 30500 Mound Road, \\ Warren, MI, USA 48090-9055 \\ Jonathan D. Hauenstein ${ }^{1,3}$ \\ Department of Mathematics, Texas AEM University, Mailstop 3368, College Station, TX, \\ USA 77843-3368 \\ Andrew J. Sommese ${ }^{1,4}$ \\ Department of Applied \& Computational Mathematics \& Statistics, University of Notre \\ Dame, Notre Dame, IN, USA 46556-4618
}

\begin{abstract}
The mobility of a mechanism is the number of degrees of freedom (DOF) with which it may move. This notion is mathematically equivalent to the dimension of the solution set of the kinematic loop equations for the mechanism. It is well known that the classical Grübler-Kutzbach formulas for mobility can be wrong for special classes of mechanisms, and even more refined treatments based on displacement groups fail to correctly predict the mobility of so-called "paradoxical" mechanisms. This article discusses how recent results from numerical algebraic geometry can be applied to the question of mechanism mobility. In particular, given an assembly configuration of a mechanism and its loop equations, a local dimension test places bounds on the mobility of the associated assembly mode. A publicly available software code makes the idea easy to apply in the kinematics domain.
\end{abstract}

Keywords: mobility, local dimension, parallel manipulators, overconstrained mechanisms, self-motion, numerical algebraic geometry

\footnotetext{
${ }^{*}$ Corresponding author

Email addresses: charles.w.wampler@gm.com (Charles W. Wampler), jhauenst@math.tamu.edu (Jonathan D. Hauenstein), sommese@nd.edu (Andrew J. Sommese) URL: http://www.nd.edu/ cwample1 (Charles W. Wampler), http://www . math.tamu.edu/ jhauenst (Jonathan D. Hauenstein), http://www.nd.edu/ sommese. (Andrew J. Sommese)

${ }^{1}$ This material is based on work supported by the National Science Foundation under Grant No. 0712910.

${ }^{2}$ Also supported by General Motors Research and Development.

${ }^{3}$ Also supported by the Fields Institute.

${ }^{4}$ Also supported by the Duncan Chair of the University of Notre Dame.
} 


\section{Introduction}

The most basic property of a mechanism is its mobility, that is, its number of degrees of freedom (DOFs). One may also speak of the mobility of a family of mechanisms: for example, one may say that planar four-bar linkages have mobility one. Such statements are properly understood to mean that almost all of the mechanisms in the family have the stated mobility, although there may be exceptions. A classical example is the family of $4 \mathrm{R}$ spatial single-loop mechanisms. Four general R-R links cannot even be assembled into a closed loop, yet planar, spherical, and Bennett four-bars all assemble with mobility one. (Delassus [7] showed that there are no other moveable four-bars.) Another notable example is the family of Stewart-Gough parallel-link (6SPU) robots, which when the leg lengths are held fixed may be considered as 6SU mechanisms. Most 6SU mechanisms are structures, that is, they have mobility zero. They can be assembled in a finite number of configurations (at most 40) and are immobile in each of these. Nevertheless, exceptional cases exist of 6SU mechanisms that have mobility one, specifically the architecturally singular Stewart-Gough platforms as classified by Karger [15] and a moveable platform found by Geiss and Schreyer [8] that is not architecturally singular. The mobilities of many families of mechanisms, particularly those described by just a list of the number of links and the kind of joints between them, submit to simple formulas, such as the GrüblerKutzbach formulas. In contrast, those families whose description includes extra geometric constraints, such as parallel or perpendicular joint axes or particular combinations of link lengths, often require a more detailed analysis.

The situation is even more complicated than just indicated, for some mechanisms have assembly modes of different mobility. The existence of such mechanisms forces one to speak of the mobility of each assembly mode rather than the mobility of the mechanism. It may even happen that two assembly modes of different mobility happen to meet, in which case the mobility of the mechanism can change at a point of intersection. Mechanisms with this property are said to be kinematotropic [26].

Kinematicians also speak of "finite mobility" and "infinitesimal mobility." An infinitesimal degree of freedom corresponds to a direction of motion that exists to first or higher differential order but does not extend to a finite motion. As we shall discuss, these degrees of freedom are intimately connected to the concept of roots that have multiplicity greater than one. All degrees of freedom, finite and infinitesimal, lie in the null-space of the Jacobian matrix for the loop equations. This article describes an extension of the Jacobian matrix, called a Macaulay matrix, which includes higher order terms that can be used to distinguish between finite and infinitesimal directions, thus arriving at the finite mobility of the mechanism. The methodology involved comes from work in numerical algebraic geometry, where the Macaulay matrix is central to a local dimension test that is used to sort solution points found by numerical continuation[1].

In short, the contribution of this article is to show how the local dimension test from numerical algebraic geometry applies to determining the mobility of 
an assembly mode of a mechanism. We describe an algorithm whose inputs are: (1) a mechanism as defined by its loop equations, (2) an assembly configuration of the mechanism, and (3) an upper limit on the order of the analysis. The output is a determination of the local mobility up to the given order. We show how the method can often be applied to a whole mechanism family. Care is taken to clarify the mathematical meaning of the computed results.

The paper begins with a short review of mobility analysis as currently conducted in the kinematics community. We then review Macaulay matrices and the local dimension test based on them. This leads to a new approach to computing mobility, which we illustrate on several examples.

\section{Mobility Analysis}

To place the current work in context, we begin with a brief review of existing methods for determining the mobility of mechanisms and mechanism families. A more detailed review of the field is available in [9].

The idea that underlies formulas for calculating mobility is basically a count of the number of variables and the number of constraint equations, the latter being the loop closure equations for a mechanism. The difference between these is a first guess at the mobility of the mechanism, as each (scalar) loop equation has the potential to reduce the mobility by one. However, this guess is only correct if each of the equations places an independent constraint on the motion. The question of independence is thus at the heart of the matter.

The need for a more refined approach than counting variables and equations is immediately apparent in the kinematics context. Consider a rigid body in three-space. Its location is described by a position and orientation, say $(p, R) \in$ $S E(3)=\mathbb{R}^{3} \times S O(3)$. Here, $S O(3)$ is the set of $3 \times 3$ rotation matrices given by

$$
S O(3)=\left\{R \in \mathbb{R}^{3 \times 3}\left|R^{T} R=I, \quad\right| R \mid=1\right\} .
$$

It is well-known that $\operatorname{dim} S O(3)=3$, but this is not immediately apparent from a count of variables and equations, as detailed next.

Example 2.1 (SO(3)). Matrix $R$ in Eq. 1 has nine entries. Due to symmetry, the matrix equation $R^{T} R=I$ is equivalent to just six scalar equations, so with the final equation $|R|=1$, there are a total of seven. If the seven equations were independent, one would have that $S O(3)$ is two dimensional $(9-7=2)$, whereas it is known to be three dimensional. The first six equations determine two sets of dimension three, the set of rotations having $|R|=1$ and the set of mirror-image rotations having $|R|=-1$. Thus the final equation, $|R|=1$, does not reduce the dimension of the set; instead, it picks out the rotations and discards their mirror images.

The simplest mobility formulas, which we refer to as Grübler-Kutzbach formulas $[10,16]$, account for the dimension of the ambient motion space of the links. A free-floating rigid body in three-space has six degrees of freedom, the 


\begin{tabular}{|c|l|}
\hline $\mathcal{F}$ & Type \\
\hline 1 & rotational, $R$; prismatic, $P$; helical/screw, $H$ \\
2 & cylindrical, $C$ \\
3 & spherical, $S$; planar, $E$ \\
\hline
\end{tabular}

Table 1: Degrees of freedom of the lower-order pairs

dimension of $S E(3)$. Thus, $\mathcal{D}=6$ is the ambient dimension for spatial mechanisms. But for planar or spherical mechanisms, the ambient dimension is $\mathcal{D}=3$ being the dimension of $\mathbb{R}^{2} \times S O(2)$ and $S O(3)$, respectively. Declaring one link as a fixed ground link, we have that a mechanism built with $N$ links has $\mathcal{D}(N-1)$ degrees of freedom before connecting the links with joints. Suppose that a two-link mechanism with a single joint of a certain type (e.g., revolute or prismatic) has $\mathcal{F}$ degrees of freedom. This implies that the joint removes $\mathcal{C}=\mathcal{D}-\mathcal{F}$ degrees of freedom, and $\mathcal{C}$ is called the degree of constraint of the joint. The Grübler-Kutzbach formulas assume that such a joint always removes $\mathcal{C}$ freedoms no matter where it is placed in a multi-link mechanism so that the resulting mobility is:

$$
D O F=\mathcal{D}(N-1)-\sum_{j \in J} \mathcal{C}_{j}=\sum_{j \in J} \mathcal{F}_{j}-\mathcal{D} \mathcal{L},
$$

where $J$ is the set of joints, $\mathcal{C}_{j}$ is the number of constraints imposed by joint $j$, $\mathcal{F}_{j}$ is the number of freedoms allowed by joint $j$, and $\mathcal{L}$ is the number of loop closures. Variants of the formulas derive from topological relations between the number of links, joints, and loops. The degrees of freedom of the basic "lower-order pair" joints and their kinematic symbols are given in Table 1.

It is believed that these formulas are correct for any general mechanism in a family described only by a list of links and their joints. That is, other than the conditions defining the ambient motion space (e.g., spherical mechanisms have only rotational joints that intersect at the origin), the parameters of the links have no other special relations. The correctness of the Grübler-Kutzbach formulas in this generic sense has never been rigorously proven, although some work in that direction may be found in [19].

Just as the spatial Grübler-Kutzbach formulas must be modified for the planar and spherical cases, more extensive modifications are needed for mechanisms that mix planar, spherical, and spatial sub-mechanisms. Displacement group methods [12] deal with these and related subgroups of $S E(3)$, such as the Schönflies subgroup $\mathbb{R}^{3} \times S O(2)$. Roughly speaking, by analyzing what subgroups are generated by the branches of a tree-like mechanism and then using knowledge of how these subgroups intersect when the branches join to form a closed-loop mechanism, the analyst may derive the mobility of many complicated mechanisms. The approach can often account for joints that are successively parallel, perpendicular, or intersecting.

Still, the displacement group approach does not account for extra degrees of freedom that arise due to special values of the link parameters. Hervé [12] 
classifies mechanisms into three categories: "trivial," for which the GrüblerKutzbach formulae suffice; "exceptional," which are not trivial but whose mobility is captured by displacement group theory; and "paradoxical," in which more complicated relationships in the link lengths, twists, and offsets affect the mobility. The classic example of a paradoxical mechanism is the Bennett spatial four-bar [4], which has mobility one due to a special relation between link lengths and link twists (with all link offsets equal to zero). Also in this class are the architecturally singular Stewart-Gough platforms previously mentioned.

A different approach to generalizing the Grübler-Kutzbach formulas depends on a technique of replacing links and joints with an equivalent polyhedral model [25]. This approach overlaps with the group theory approach in the ability to deal with some exceptional mechanisms. It has also been used to successfully explain the mobility of certain special mechanisms that contain scissor linkage sub-mechanisms.

Yet another approach, closely related to the current contribution, is to compute mobility as the corank of the Jacobian matrix evaluated at an assembly configuration of the mechanism $[20,21,28]$. Since these approaches directly use the differentials of the loop equations, they correctly predict the mobility of many paradoxical mechanisms provided that one knows at least one general assembly configuration. While this general approach is useful, it has limitations. In this article, we elucidate these limitations and give a methodology that moves beyond them.

All mechanisms built with lower-order pair joints of type $P, R, C, E$, or $S$, that is, excepting helical type $H$, are algebraic. That is to say that the loop equations for the mechanism can be written as a system of polynomial equations. Examples of algebraic formulations for kinematics can be found in [13]. Potentially, the equations can be solved using symbolic methods from computer algebra. Once a Grobner basis has been found, the dimension of the solution set can be determined by computing a Hilbert function[14].

Numerical algebraic geometry [22, 23], which is based on continuation methods, is able to solve larger systems than symbolic methods. The strength of this approach is that it is fully automatic and does not require foreknowledge of an assembly configuration. The downside is that the process of finding all solution components can be expensive, although the speed of the algorithms involved has improved remarkably in recent years [11]. These methods compute other characteristics of the motion, such as its degree, that may not be of interest in early studies, so it may be valuable to have a less expensive approach that computes the minimal information required to determine mobility. In fact, the latest algorithms in numerical algebraic geometry use a local dimension test, described in more detail below, to sort the solution points found by continuation. In this paper, we assume we start with a mechanism in a known assembly configuration, in which case a slight variant of the local dimension test can be employed to obtain information about the mobility of the associated assembly mode.

The local dimension test is based on the partial derivatives of the loop equations at the given assembly configuration. As such, it is a purely local analysis 
and does not depend on the global properties of polynomial systems (finite degree, in particular). The approach only requires that the loop equations be complex analytic, a condition that is satisfied by helical joints (type $H$ ) as well as all the algebraic joints $(P, R, C, E, S)$.

The full situation is best explained by a pathway that temporarily leads us outside of kinematics into the realm of general analytic geometry.

\section{Dimension and Local Dimension}

To discuss mobility in kinematics, it is helpful to adopt concepts from complex analytic geometry. More detail on the ideas outlined here can be found in [23, § A.1].

The class of functions of interest to us is the class of analytic functions, also known as holomorphic functions, the functions which in any sufficiently small open neighborhood of a point $p$ in the domain of definition can be expressed as an absolutely convergent power series with complex coefficients centered at the point $p$. These functions include all polynomials as well as the elementary trigonometric and exponential functions. This covers all the kinds of functions one finds in kinematic loop closure equations.

A complex analytic set is the zero set of $n$ analytic functions in $N$ unknowns, $f(x), f: \mathbb{C}^{N} \rightarrow \mathbb{C}^{n}$. In kinematics, we generally are only interested in the real solution set

$$
\mathcal{V}_{\mathbb{R}}(f)=\left\{x \in \mathbb{R}^{N} \mid f(x)=0\right\},
$$

but since the reals are contained within the complexes, it is helpful to first consider the complex solution set

$$
\mathcal{V}_{\mathbb{C}}(f)=\left\{x \in \mathbb{C}^{N} \mid f(x)=0\right\} .
$$

The algebraic completeness of the complex field makes some calculations easier, so we consider first the dimension of $\mathcal{V}_{\mathbb{C}}(f)$ and then consider its real part. When $Z$ is a complex analytic set, we sometimes write $\operatorname{real}(Z)$ to denote the real points in $Z$. Clearly,

$$
\operatorname{real}\left(\mathcal{V}_{\mathbb{R}}(f)\right)=\mathcal{V}_{\mathbb{R}}(f)=\operatorname{real}\left(\mathcal{V}_{\mathbb{C}}(f)\right)
$$

We elaborate on the relationship between real and complex dimension in $\S 4$.

Informally, the dimension of an analytic set is the minimum number of coordinates needed to generically specify points on the set uniquely and continuously. A more rigorous definition of dimension uses the concept of biholomorphic mappings. A holomorphic mapping, say $\phi: X \rightarrow Y$, from complex analytic set $X$ to complex analytic set $Y$ is a mapping given by analytic functions. The mapping is called biholomorphic if it has a holomorphic inverse, that is, a holomorphic mapping $\psi: Y \rightarrow X$ such that $\psi(\phi(x))=x$ for all $x$ in an open subset of $X$ and $\phi(\psi(y))=y$ for all $y$ in an open subset of $Y$. If there exists a biholomorphic mapping from $X$ to $Y$, then we say that $X$ is biholomorphic to $Y$. We skip 
some of the technical details here, but all we need is for the biholomorphism to hold on a local neighborhood of a point.

Let $Z \subset \mathbb{C}^{N}$ be an analytic set and let $z \in Z$ be a point. If a neighborhood of $Z$ that contains $z$ is biholomorphic to a neighborhood of a point in $\mathbb{C}^{k}$, then we say that $Z$ is smooth at $z$ and the local dimension of $Z$ at $z$ is $k$, written $\operatorname{dim}_{z} Z=k$. The idea is that the coordinate axes in $\mathbb{C}^{k}$ provide a local coordinate patch for $Z$ in the neighborhood of $z$. As one would expect, the dimension of $\mathbb{C}^{k}$ at any point is $k$, since $\mathbb{C}^{k}$ is biholomorphic to itself under the identity map. Notice that $\mathbb{C}^{k}$ has real dimension $2 k$ due to the fact that each complex coordinate direction has a real and an imaginary part. Assigning the complex dimension $k$ to $\mathbb{C}^{k}$ has the agreeable property that the dimension of the set of real points in $\mathbb{C}^{k}$, which is just $\mathbb{R}^{k}$, is also a $k$ dimensional set. Thus, for Euclidean sets, the real and complex dimensions are the same. This is not the case for all the sets of interest, as explained further in $\S 4$.

The smooth points of a complex analytic set $Z$ are denoted $Z_{\text {reg }}$ (they are also known as regular points), and their complement in $Z$ is called the singular set of $Z$, written $Z_{\text {sing }}=Z \backslash Z_{\text {reg. }}$. The definition of local dimension just given only applies at smooth points. Fortunately, it can be shown that the set $Z_{\text {reg }}$ is open and dense in $Z$, so any singular point has neighbors that are smooth. So, we may define the local dimension of $Z$ at a singular point $z$ as the maximum of the local dimension of its smooth neighbors in $Z$.

Example 3.1. Suppose $f(x, y, z)=\{x z, y z\}$, so $Z=\mathcal{V}_{\mathbb{C}}(f)=Z_{1} \cup Z_{2}$ where $Z_{1}$ is the line $\mathcal{V}_{\mathbb{C}}(x, y)$ and $Z_{2}$ is the plane $\mathcal{V}_{\mathbb{C}}(z)$. Away from the origin, a biholomorphism between a local neighborhood of $Z_{1}$ and a local neighborhood of $\mathbb{C}$ is given by the pair of maps

$$
\phi_{1}:(x, y, z) \mapsto(z), \quad \psi_{1}(t):(z) \mapsto(0,0, z) .
$$

[To check the biholomorphism, simply observe that for any point $w \in Z_{1}$, say $w=(0,0, \alpha), \psi_{1}\left(\phi_{1}(w)\right)=w$ and for any point $\alpha \in \mathbb{C}, \phi_{1}\left(\psi_{1}(\alpha)\right)=\alpha$.] Similarly, away from the origin, we have a biholomorphism between $Z_{2}$ and $\mathbb{C}^{2}$ given by the maps

$$
\phi_{2}:(x, y, z) \mapsto(x, y), \quad \psi_{2}:(x, y) \mapsto(x, y, 0)
$$

Neither of these pairs of maps covers all the points in the neighborhood of the origin, $(0,0,0)$, where the two components $Z_{1}$ and $Z_{2}$ meet. The origin is singular while all other points of $Z$ are smooth. The local dimension at two of the smooth points is: $\operatorname{dim}_{(0,0,1)} Z=1$ and $\operatorname{dim}_{(1,1,0)} Z=2$. The origin has neighbors in $Z_{1}$ and in $Z_{2}$, so the local dimension at the origin is the greater of these two: $\operatorname{dim}_{(0,0,0)} Z=2$. Figure 1 illustrates a related example, where $Z$ consists of a surface and a curve, each nonlinear.

In Example 3.1, it is easy to write down maps that establish the local dimension at smooth points, but in general, this is not so simple. However, there is one frequently occurring situation where local dimension is easy to establish, 


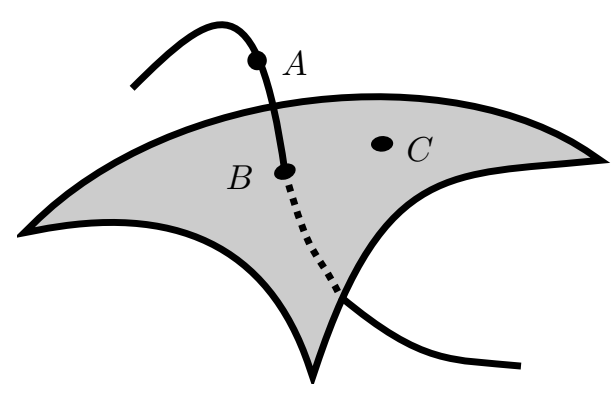

Figure 1: A set $Z$ with local dimensions: $\operatorname{dim}_{A} Z=1, \operatorname{dim}_{B} Z=\operatorname{dim}_{C} Z=2$.

as stated in the following well-known proposition. For a system of $m$ analytic functions in $N$ variables, $f\left(x_{1}, \ldots, x_{N}\right)=\left\{f_{1}, \ldots, f_{m}\right\}: \mathbb{C}^{N} \rightarrow \mathbb{C}^{m}$, we denote the $m \times N$ Jacobian matrix of $f$ as $d f$, having elements $d f_{i j}=\partial f_{i} / \partial x_{j}$. The dimension of the right null space of $d f$ is called the corank of $d f$ :

$$
\operatorname{corank}(d f)=N-\operatorname{rank}(d f) .
$$

Proposition 3.1. Suppose $Z \subset \mathbb{C}^{N}$ is given by $m \leq N$ functions, $f: \mathbb{C}^{N} \rightarrow$ $\mathbb{C}^{m}, Z=\mathcal{V}_{\mathbb{C}}(f)=\mathcal{V}_{\mathbb{C}}\left(f_{1}, \ldots, f_{m}\right)$, and suppose that the Jacobian matrix of $f$ is full rank at $z \in Z$, i.e., $\operatorname{rank}(d f(z))=m$. Then, $\operatorname{dim}_{z} Z=N-m=\operatorname{corank}(d f)$.

Proof. If necessary, re-order the variables such that the trailing $m \times m$ block of $d f$ is full rank, say $d f=\left[\begin{array}{ll}J_{1} & J_{2}\end{array}\right], J_{2} \in \mathbb{C}^{m \times m}, \operatorname{rank}\left(J_{2}\right)=m$. Subdivide the variables in a compatible way: $x=(u, v), u=\left(x_{1}, \ldots, x_{N-m}\right)$, $v=\left(x_{N-m+1}, \ldots, x_{N}\right)$. Then, the map $\phi: Z \rightarrow \mathbb{C}^{N-m}$ given by $(u, v) \mapsto(u)$ has an inverse $\psi$ in the neighborhood of $z=\left(u_{0}, v_{0}\right)$ that is approximated to first order as $(u) \mapsto\left(u, v_{0}-J_{2}^{-1} J_{1}\left(u-u_{0}\right)\right)$. While this is just an approximation, the implicit function theorem says that an exact holomorphic inverse exists on an open neighborhood of $\phi(z)$.

Local dimension is defined in terms of the existence of biholomorphic maps, but does not give a prescription for constructing such maps. The difficulties arise when the conditions of Proposition 3.1 are not satisfied; that is, when the Jacobian matrix of the functions defining an analytic set is not full rank. The following two examples illustrate that when the count of excess variables, $N-m$, is not equal to the Jacobian corank, $\operatorname{corank}(d f)$, neither can be trusted to give the proper dimension.

Example 3.2 ( $\mathrm{SO}(3)$ continued.). In Example 2.1, we saw that $S O(3)$ is given by $m=7$ equations in $N=9$ variables. The Jacobian of the equations at any point of $S O(3)$ has only rank 6 , so $\operatorname{corank}(d f)=3$. In this case,

$$
2=N-m<\operatorname{corank}(d f)=3=\operatorname{dim} S O(3)
$$


Example 3.3. Let $f(x, y)=x^{2}$, so that $Z=\mathcal{V}_{\mathbb{C}}(f)=\left\{(x, y) \in \mathbb{C}^{2} \mid(x, y)=\right.$ $(0, t), t \in \mathbb{C}\}$. In other words, $Z$ is the $y$-axis in the $(x, y)$-plane. Clearly, $Z$ is one-dimensional, but the Jacobian at any point on $Z$ is $\left[\begin{array}{ll}0 & 0\end{array}\right]$ :

$$
\operatorname{dim} Z=1=N-m<\operatorname{corank}(d f)=2 .
$$

Although neither measure necessarily gives the correct local dimension, they do give bounds on it. As usual, let $Z=\mathcal{V}_{\mathbb{C}}(f)=\mathcal{V}_{\mathbb{C}}\left(f_{1}, \ldots, f_{m}\right), f: \mathbb{C}^{N} \rightarrow \mathbb{C}^{m}$. As in $[23, \S 13.4]$, we define $\operatorname{rank} f$ as the rank of its Jacobian at a generic point $z^{*} \in \mathbb{C}^{N}$, that is, $\operatorname{rank} f:=\operatorname{rank} d f\left(z^{*}\right)$. Then, we have

$$
N-m \leq N-\operatorname{rank} f \leq \operatorname{dim}_{z} Z \leq \operatorname{corank} d f(z) .
$$

Example 3.1 illustrates that an analytic set may break up into sets with different properties, in that case, a one-dimensional line and a two-dimensional plane. This kind of break-up also applies locally in the neighborhood of a point on an analytic set. More precisely, if one removes the singular points of a complex analytic set, the remaining smooth points break up into connected irreducible components. The irreducible components meet only at singularities. The local and global irreducible break-ups do not necessarily agree. For example, an irreducible curve may cross itself (think of a curve with a section in the shape of the Greek letter $\alpha$ ). At the point of crossing, the local irreducible decomposition has two pieces, local segments of the curve, while the whole curve is a single piece in the global irreducible decomposition.

An important distinction is to be drawn between reduced and nonreduced components. Suppose that $z^{*} \in Z_{\text {reg }}$ is a smooth point of $Z$. If $\operatorname{dim}_{z^{*}} Z=$ corank $d f\left(z^{*}\right)$, then we say that $z^{*}$ is on a reduced component of $Z$. Conversely, any component whose dimension is less than the corank of the Jacobian at its smooth points is called a nonreduced component. Example 3.3 gives an example of a nonreduced component: $\mathcal{V}_{\mathbb{C}}\left(x^{2}\right)$ and $\mathcal{V}_{\mathbb{C}}(x)$ define the same set of points, but only $\mathcal{V}_{\mathbb{C}}(x)$ is reduced. As we shall see, the dimensionality of nonreduced components can be computed by evaluating higher order derivatives of $f$.

\section{Complex Dimension and Real Dimension}

Up to this point, we have not distinguished between real and complex dimension. While in many cases these are the same, they may differ. A few examples will help set ideas. First, consider a complex line in $\mathbb{C}^{2}$, say for example, $\mathcal{V}_{\mathbb{C}}(x-y)$. This can be parameterized as $(x, y)=(t, t), t \in \mathbb{C}$, and hence has complex dimension one. This is equivalent to $(x, y)=(a+b i, a+b i)$, $a, b \in \mathbb{R}$, where $i=\sqrt{-1}$, so we may say that over the complexes $\mathcal{V}_{\mathbb{C}}(x-y)$ is two real dimensional. However, the real points of this set are just $\mathcal{V}_{\mathbb{R}}(x-$ $y)=\{(x, y)=(a, a), a \in \mathbb{R}\}$, a one dimensional set. Thus, we may write $\operatorname{dim} \mathcal{V}_{\mathbb{R}}(x-y)=\operatorname{dim} \mathcal{V}_{\mathbb{C}}(x-y)=1$, where it is understood that the dimensions being equated are the real dimensionality of the real set and the complex dimensionality of the complex set, respectively. 
Consider more generally any system of linear equations with real coefficients, say $A x-b=0, A \in \mathbb{R}^{m \times n}, x \in \mathbb{C}^{n}, b \in \mathbb{R}^{m}$. The solution set of the linear system can be written as $x_{p}+x_{h}$, where $x_{p}$ is a particular solution and $x_{h}$ is any vector in the null space of $A, x_{h} \in\left\{v \in \mathbb{R}^{n} \mid A v=0\right\}$. Since $A$ is real, the null space of $A$ is given by a set of real basis vectors. For example, the particular solution and the basis vectors may be computed by Gaussian row reduction, and since $A$ and $b$ are real, all the computed quantities remain real throughout the computation. By this reasoning, we see that the real and complex dimensions of any linear space given by real equations must be the same.

The local dimension at a smooth point of a reduced component is by definition equal to the corank of the Jacobian at the point. If the system of functions, $f$, that define the component are such that the Jacobian matrix is real at any real point then the real and complex local dimensions at a real smooth point must be equal: $\operatorname{dim}_{z} \mathcal{V}_{\mathbb{C}}(f)=\operatorname{dim}_{z} \mathcal{V}_{\mathbb{R}}(f), z$ real, smooth. For example, this will be true for any smooth point on a reduced component of a polynomial with all real coefficients.

The equality of the real and complex dimensions of a complex analytic component can fail for several reasons. One case is when there are no real points in the complex set. This occurs, for example, in the case of $x^{2}+y^{2}+1=0$, which describes a complex circle that has no real points (for real $(x, y), x^{2}+y^{2}$ can never be negative). This case is irrelevant to the remainder of this paper, because we assume we are given a real point of the solution set (a real assembly configuration of a mechanism), hence the subset of real points in the complex set cannot be empty.

The equality of the real and complex dimensions may also fail at a singular point.

Example 4.1. A simple example is $x^{2}+y^{2}=0$, which describes a one complex dimensional set, namely the pair of lines $\mathcal{V}_{\mathbb{C}}(x+i y)$ and $\mathcal{V}_{\mathbb{C}}(x-i y)$, whereas the real solution set is a single point, $\mathcal{V}_{\mathbb{R}}\left(x^{2}+y^{2}\right)=(0,0)$. Notice that the real point occurs where the two complex lines intersect.

Example 4.2. A more interesting example is the set $Z=\mathcal{V}_{\mathbb{C}}\left(y^{2}-x^{2}(x-1)\right)$, which is a single irreducible complex curve: $\operatorname{dim} Z=1$. In contrast, the real points of $Z$ consist of a curve $y= \pm \sqrt{x^{2}(x-1)}, x \geq 1$, and the isolated real point $(0,0)$.

These two examples are related, as near the origin $y^{2}-x^{2}(x-1) \approx x^{2}+y^{2}$. In Example 4.2, the origin is a point where the complex cubic curve crosses itself.

While singularity is necessary for the complex and real local dimension at a real point to differ, it is not sufficient. The origin is a singular point of $x^{2}-y^{2}$, which lies at the crossing of two lines $\mathbf{V}(x-y)$ and $\mathbf{V}(x+y)$. In this case, the real and complex local dimensions at the origin are both equal to 1 .

In higher dimensions, the set of real points of a complex analytic set of dimension $k$ can be empty or it can have any dimension less than or equal to $k$. For example, $x^{2}+y^{2}+z^{2}=0$ defines a surface in $\mathbb{C}^{3}$ that contains only an isolated real point, the origin. A complex surface that contains only one real 
curve is defined by $\left(x^{2}+y^{2}-1\right)^{2}+z^{2}=0$. (The real curve is a unit circle in the $z=0$ plane.)

The situation can be summarized as follows:

1. The dimension of the set of real points in a complex analytic set is less than or equal to the complex dimension of the set. This also applies to local dimension. That is, for any algebraic set $Z \subset \mathbb{C}^{N}$, and for any real point $z \in Z$,

$$
\operatorname{dim} \operatorname{real}(Z) \leq \operatorname{dim} Z, \quad \text { and } \quad \operatorname{dim}_{z} \operatorname{real}(Z) \leq \operatorname{dim}_{z} Z .
$$

2. If $z$ is a regular point of $Z$, then $\operatorname{dim}_{z} \operatorname{real}(Z)=\operatorname{dim}_{z} Z$.

\section{Multiplicity and Local Dimension}

The local dimension of an analytic set is closely tied to the concept of multiplicity. Consider the equations in one variable $x(x-1)=0$ and $x^{2}(x-1)=0$. For either equation, the solution set is just two points, the origin and $x=1$, that is, $\mathcal{V}_{\mathbb{C}}(x(x-1))=\mathcal{V}_{\mathbb{C}}\left(x^{2}(x-1)\right)=\{0,1\}$, but the character of the solutions differ. Both have $x=1$ as a simple root, but $x=0$ appears as a simple root of $x(x-1)=0$ and appears as a double root of $x^{2}(x-1)$, that is, it has multiplicity one and two in the two cases, respectively. Multiple roots tend to be more sensitive to perturbations than simple roots or, said another way, allowing some small variability away from an exact equality, the multiple root has more leeway to move than the simple one. In our example, for small real positive $\epsilon$, the inequality $|x(x-1)|<\epsilon$ admits all real $x$ near zero in the range of approximately $[-\epsilon, \epsilon]$ while for $\left|x^{2}(x-1)\right|<\epsilon$ this expands to approximately the range $[-\sqrt{\epsilon}, \sqrt{\epsilon}]$. This is a real effect that is observed in mechanical systems: mechanisms in a configuration associated with a multiple root tend to be "loose," admitting small motions in the neighborhood of the root. As mentioned earlier, these motions are often referred to as infinitesimal degrees of freedom. A more concrete example is a three-bar planar $R R R$ linkage (a geometric triangle) with the sum of the two shorter link lengths equal to the third link length. Due to link elasticity and joint clearances, such a degenerate triangle will allow small deflections.

As the order of a multiple root increases, so does its sensitivity; e.g., $x^{3}=$ 0 is more sensitive to perturbation than $x^{2}=0$, etc. The functions $x, x^{2}$, and $x^{3}$ have zero, one, and two derivatives, resp., that vanish at the origin. The identically zero function $f(x):=0$, which admits all $x \in \mathbb{C}$ as a onedimensional solution set, may be thought of as having a root at the origin of infinite multiplicity: all the derivatives $d^{k} f / d x^{k}, k=1,2, \ldots, \infty$, vanish at the origin. This rough idea can be made into a precise mathematical theory that relates the number of vanishing derivatives to multiplicity and dimension.

The relation between vanishing derivatives, multiplicity, and dimension is easiest to understand through a construction we call a Macaulay matrix [18], recently reintroduced in a modern computational context by Dayton and Zeng [6]. This matrix organizes the analysis of higher derivatives. The following 
multidegree notation is useful. For $\alpha=\left(\alpha_{1}, \ldots, \alpha_{N}\right) \in \mathbb{Z}_{\geq 0}^{N}$, i.e., a vector of $N$ nonnegative integers, and $x=\left(x_{1}, \ldots, x_{N}\right)$, let

$$
\begin{gathered}
|\alpha|:=\alpha_{1}+\cdots+\alpha_{N}, \\
\alpha !:=\alpha_{1} ! \cdots \alpha_{N} !, \\
x^{\alpha}:=x_{1}^{\alpha_{1}} \cdots x_{N}^{\alpha_{N}}, \\
D^{\alpha} f:=\frac{1}{\alpha !} \frac{\partial^{|\alpha|} f}{\partial x_{1}^{\alpha_{1}} \cdots \partial x_{N}^{\alpha_{N}}} .
\end{gathered}
$$

With these notations, the $d$-th order Macaulay matrix for a system of functions $f(x)=\left(f_{1}(x), \ldots, f_{m}(x)\right)$ has columns indexed by $\alpha$ and rows indexed by $(\beta, j)$ with elements

$$
\mathcal{M}_{d}(z)=\left[D^{\alpha}\left(x^{\beta} f_{j}\right)(z)\right],
$$

where $\alpha$ ranges over all possibilities with $|\alpha| \leq d, \beta$ ranges over all $|\beta| \leq$ $\max (0, d-1)$, and $1 \leq j \leq m$. Notice that the derivatives, $D^{\alpha}$, have been defined such that if $f$ is a polynomial, then $D^{\alpha}(f)(0)$ is the coefficient of the monomial $x^{\alpha}$. This observation makes it easy to write down examples like the following one.

Example 5.1. For $f=\left\{y-x^{2}, x^{3}\right\}$, the third-order Macaulay matrix evaluated at $(0,0)$ is

$\mathcal{M}_{3}(0)=\left(\begin{array}{r|ccccccccccc} & (0,0) & (1,0) & (0,1) & (2,0) & (1,1) & (0,2) & (3,0) & (2,1) & (1,2) & (0,3) \\ \hline f_{1} & 0 & 0 & 1 & -1 & 0 & 0 & 0 & 0 & 0 & 0 \\ f_{2} & 0 & 0 & 0 & 0 & 0 & 0 & 1 & 0 & 0 & 0 \\ x f_{1} & 0 & 0 & 0 & 0 & 1 & 0 & -1 & 0 & 0 & 0 \\ y f_{1} & 0 & 0 & 0 & 0 & 0 & 1 & 0 & -1 & 0 & 0 \\ x f_{2} & 0 & 0 & 0 & 0 & 0 & 0 & 0 & 0 & 0 & 0 \\ y f_{2} & 0 & 0 & 0 & 0 & 0 & 0 & 0 & 0 & 0 & 0 \\ x^{2} f_{1} & 0 & 0 & 0 & 0 & 0 & 0 & 0 & 1 & 0 & 0 \\ x y f_{1} & 0 & 0 & 0 & 0 & 0 & 0 & 0 & 0 & 1 & 0 \\ y^{2} f_{1} & 0 & 0 & 0 & 0 & 0 & 0 & 0 & 0 & 0 & 1 \\ x^{2} f_{2} & 0 & 0 & 0 & 0 & 0 & 0 & 0 & 0 & 0 & 0 \\ x y f_{2} & 0 & 0 & 0 & 0 & 0 & 0 & 0 & 0 & 0 & 0 \\ y^{2} f_{2} & 0 & 0 & 0 & 0 & 0 & 0 & 0 & 0 & 0 & 0\end{array}\right)$.

The lines in the matrix show how $\mathcal{M}_{0}(0), \mathcal{M}_{1}(0)$, and $\mathcal{M}_{2}(0)$ appear as submatrices of $\mathcal{M}_{3}(0)$.

In some presentations of the Macaulay matrix, e.g., [6], the elements are defined as

$$
\mathcal{M}_{d}(z)=D^{\alpha}\left((x-z)^{\beta} f_{j}\right)(z) .
$$

This is interchangeable with the definition above, since we will only be concerned with the column space of the matrix, and it can be seen that the matrices for the two definitions differ only by premultiplication by a nonsingular matrix. 
For a point $z \in \mathbb{C}^{N}$, let $c_{d}(z)$ be the column corank of $\mathcal{M}_{d}(z)$, that is,

$$
c_{d}(z)=\left(\begin{array}{c}
d+N \\
d
\end{array}\right)-\operatorname{rank} \mathcal{M}_{d}(z),
$$

where the binomial coefficient is the number of columns of $\mathcal{M}_{d}(z)$. In Example 5.1, this gives $c_{0}(0)=1, c_{1}(0)=2, c_{2}(0)=c_{3}(0)=3$. (The sequence $c_{d}(z)$ determines a Hilbert function, $\mathcal{H}(d)=c_{d}(z)-c_{d-1}(z)$, a concept often used in algebraic geometry, but we find $c_{d}(z)$ more useful here.)

The growth rate of the sequence of coranks, $c_{d}(z), d=0,1,2, \ldots$, reveals the local dimension at $z$. If $z$ is an isolated solution (i.e., dimension $=0$ ), then the coranks reveal its multiplicity. Note that $c_{d}(z)$ is at most $\left(\begin{array}{c}d+N \\ d\end{array}\right)$, which is a polynomial of degree $N$ in $d$. The facts stated in the following proposition are well known; see $[6][5, \S 9.3]$ for discussions of the algebraic case.

Proposition 5.1. For any $z \in \mathcal{V}_{\mathbb{C}}(f)$,

1. The coranks $c_{d}(z)$ monotonically increase with increasing $d$.

2. If, and only if, the local dimension at $z$ is $k$, i.e., $\operatorname{dim}_{z} \mathcal{V}_{\mathbb{C}}(f)=k$, the asymptotic growth rate of $c_{d}(z)$ as a function of $d$ is $\mathcal{O}\left(d^{k}\right)$.

3. If for some $\hat{d}, c_{\hat{d}}(z)=c_{\hat{d}+1}(z)$, then $c_{d}(z)=c_{\hat{d}}(z)$ for all $d \geq \hat{d}$ and $z$ is an isolated point in $\mathcal{V}_{\mathbb{C}}(f)$.

In the case that item 3 applies, let $d^{*}$ be the minimum value of $d$ such that $c_{d}(z)=c_{d+1}(z)$. We say that $c_{d}(z)$ stabilizes at $d^{*}$. Moreover, we call $d^{*}$ the depth and $\mu(z, f)=c_{d^{*}}(z)$ the multiplicity of $z$ as a solution of $f$. It is easy to see that this notion of multiplicity agrees with the usual multiplicity of a root of a function in one variable.

Example 5.2. Consider Example 5.1. The sequence for $c_{d}(0)$ is $1,2,3,3, \ldots$ Accordingly, the origin is isolated with multiplicity 3 and depth 2.

\section{Local Dimension Testing}

A test for local dimension could be based on Proposition 5.1, item 2 by computing the sequence $c_{d}(z), d=0,1,2, \ldots$ and analyzing its growth rate. However, this tends to be prohibitively costly because the size of the Macaulay matrices grows quickly and one must carry out the computation far enough in $d$ so that the asymptotic rate dominates. This is especially problematic considering that there is no termination condition to signal when $d$ is large enough. Large matrix rank tests become doubly expensive because higher precision arithmetic is needed to overcome numerical conditioning troubles.

It is useful to consider the effect of appending extra random linear equations to the original ones. For a complex analytic system $f: \mathbb{C}^{N} \rightarrow \mathbb{C}^{m}$, we wish to consider the sliced system

$$
\mathcal{S}(f, k)=(f(x), A(x-z)), \quad A \in \mathbb{C}^{k \times N}, A \text { generic. }
$$

A more manageable local dimension test, presented in [1] follows from Proposition 5.1, item 3 and the following observation. 
Proposition 6.1. Let $Z=\mathcal{V}_{\mathbb{C}}(f) \subset \mathbb{C}^{N}$, for complex analytic system $f: \mathbb{C}^{N} \rightarrow$ $\mathbb{C}^{m}$. Let $z \in Z$ be a point in $Z$. For generic $A \in \mathbb{C}^{k \times N}$, the complex analytic set $Z^{\prime}=\mathcal{V}_{\mathbb{C}}(f(x), A(x-z))$ has local dimension

$$
\operatorname{dim}_{z} Z^{\prime}=\max \left(0, \operatorname{dim}_{z} Z-k\right) .
$$

or more tersely stated

$$
\operatorname{dim}_{z} \mathcal{V}_{\mathbb{C}}(\mathcal{S}(f, k))=\max \left(0, \operatorname{dim}_{z} \mathcal{V}_{\mathbb{C}}(f)-k\right) .
$$

By "generic," we mean that although there may exist a proper complex analytic subset of $\mathbb{C}^{k \times N}$ where the relation fails, $A$ avoids it. This will be true with probability 1 if $A$ is chosen at random.

In $\S 5$, we defined the multiplicity of an isolated point. We may define the multiplicity of a point $z$ on a positive dimensional set $Z=\mathcal{V}_{\mathbb{C}}(f)$ as

$$
\mu(z, f):=\mu\left(z, \mathcal{S}\left(f, \operatorname{dim}_{z} Z\right)\right) .
$$

That is, we append just enough linear equations through $z$ to slice $Z$ down to a point and define multiplicity in terms of the multiplicity of the newly isolated point. If we add even more slices, then it is clear that the coranks of the Macaulay matrices can only decrease, so we have

$$
\begin{array}{ll}
\mu(z, \mathcal{S}(f, k))=\mu(z, f), & k \leq \operatorname{dim}_{z} Z, \\
\mu(z, \mathcal{S}(f, k)) \leq \mu(z, f), & k>\operatorname{dim}_{z} Z .
\end{array}
$$

To simplify notation, we will write $c_{k, d}(z)$ to be $c_{d}(z)$ for $\mathcal{S}(f, k)$. Combining Proposition 5.1, item 3, with Proposition 6.1, we see that $c_{k, d}(z)$ stabilizes if, and only if, $\operatorname{dim}_{z} Z \leq k$. Consequently, $\operatorname{dim}_{z} Z$ is equal to the smallest $k$ for which the sequence $c_{k, d}(z)$ stabilizes. As long as $k \geq \operatorname{dim}_{z} Z$, we have a termination condition, namely $c_{k, d}(z)=c_{k, d+1}(z)$, which limits the computational cost of the test for large $k$. However, we do not know the correct dimension until we observe that $c_{\hat{k}, d}(z)$ stabilizes for some $\hat{k}$ but not for $\hat{k}-1$, in which case we know that $\operatorname{dim}_{z} Z=\hat{k}$.

We have the problem of establishing a stopping criterion for deciding that the sequence $c_{k, d}(z)$ for $k=\operatorname{dim}_{z} Z-1$ does not stabilize. In [1], this is provided by foreknowledge of a bound, say $\hat{\mu}$, on the multiplicity of $z$. As soon as the sequence exceeds $\hat{\mu}$, stabilization cannot occur and computation can stop. In the context of numerical continuation algorithms considered in [1], the system $f(x)$ is polynomial, and the multiplicity bound is a count of the number of paths that approach $z$ in a polynomial homotopy.

In the applications we treat here, we do not assume that we have a multiplicity bound; we only assume that an assembly configuration of a mechanism has been given. In this case, our conclusions must be weaker. To guarantee that our procedure terminates, we instead provide an artificial limit for the depth. That is, we ask the algorithm to investigate the local dimension "up to depth $\hat{d}$." For a given $k$, if $c_{k, \hat{d}-1}(z)<c_{k, \hat{d}}(z)$, we may conclude only that

$$
\text { either } \operatorname{dim}_{z} Z>k \text {, or } \mu(z, f) \geq c_{k, \hat{d}}(z) \text {. }
$$


Conversely, when the sequence does stabilize before $\hat{d}$, we know definitively that

$$
\operatorname{dim}_{z} Z \leq k
$$

We define the $\hat{d}$-bounded local dimension, written $\operatorname{dim}_{z, \hat{d}} Z$, to be the smallest $k$ that gives a stabilized $c_{k, d}(z)$ with depth less than $\hat{d}$. We have the following relations:

$$
\begin{gathered}
\lim _{\hat{d} \rightarrow \infty} \operatorname{dim}_{z, \hat{d}} Z=\operatorname{dim}_{z} Z, \\
\operatorname{dim}_{z} Z \leq \operatorname{dim}_{z, \hat{d}+1} Z \leq \operatorname{dim}_{z, \hat{d}} Z
\end{gathered}
$$

The 1-bounded local dimension, $\operatorname{dim}_{z, 1} Z$, is the corank of the Jacobian matrix, which as noted earlier was used for mobility analysis in [20,21, 28]. Relation 15 shows that this is an upper bound on the mobility; one may establish tighter bounds by considering more derivatives to find the $\hat{d}$-bounded local dimension for $d>1$.

Relations 3,14, 15 are precise statements that may be applied to the idealized mathematical model of a mechanism. Without extra information that limits the possible multiplicity, this is all that can be concluded about local dimensionality from knowing the ranks of the Macaulay matrices. In practice though, this may tell all one needs to know, because there may not be a significant practical difference between a true finite degree of freedom and an infinitesimal one of high multiplicity. A real mechanism, having link elasticity and non-ideal joints, will display a sensible freedom to move in either case. Thus, testing $z$ up to a high enough depth $\hat{d}$ for all possible dimensions provides good evidence about the actual degrees of freedom.

\section{Mechanism Families}

To this point, we have only considered a single mechanism whose loop equations are written as a system of $n$ analytic equations in $N$ variables, say $f(x)=0$, $f: \mathbb{C}^{N} \rightarrow \mathbb{C}^{n}$. A family of mechanisms is parameterized by its link parameters, say $q \in Q \subset \mathbb{C}^{m}$, where $Q$ is an algebraic set given by a polynomial system $g(q)=0$, so that the loop equations become $f(x, q)=0, f: \mathbb{C}^{N} \times \mathbb{C}^{m} \rightarrow \mathbb{C}^{n}$. For example, $g(q)=0$ might describe the Bennett conditions on the D-H parameters of a $4 \mathrm{R}$ linkage, in which case, $Q$ describes the set of all Bennett four-bars. We assume that $Q$ is irreducible, that is, it is a connected set that cannot be properly subdivided into a finite number of smaller algebraic subsets; otherwise in the reducible case, we consider each irreducible component of $Q$ to be its own family. Each point in $Q$ is a particular mechanism. The mobility of almost all mechanisms in a family is equal and can be found by determining the mobility of a randomly selected (generic) mechanism $q^{*} \in Q$, that is, by analyzing the solution set of $f\left(x, q^{*}\right)=0$. This set may also factor into more than one irreducible piece, and as we have seen in earlier examples, the dimensions of the pieces are not necessarily all the same. The number of irreducible components 
of each dimension is the same for generic points in $Q$, while for some special points lying on a proper analytic subset of $Q$, some components may disappear to infinity and some may increase in dimension (never decrease).

The degrees of freedom of a mechanism are the same as the dimension of its solution set, and different irreducible components of the solution set correspond to assembly modes of the mechanism. If we wish to apply the local dimension test to say something about the degrees of freedom of general members of a mechanism family, then we need a general assembly configuration in each assembly mode of interest. Short of applying numerical algebraic geometry (continuation) or a symbolic decomposition method to find the irreducible decomposition of the solution set, one might not be able to produce such general assembly configurations. Nevertheless, it often happens that one does have an example assembly configuration that parameterizes across a whole mechanism family. We can then pick one of these randomly and perform a local dimension test on it to learn something about the whole family. We illustrate this in $\S 9.1$.

\section{Implementation}

The software package Bertini [2], which solves systems of polynomial equations using continuation, uses a local dimension test [1] in culling out unwanted points that land on positive dimensional sets. We have adapted that test for the current purpose; the result is an algorithm, LocalDimFinder, available at the Bertini website [2]. The code, as detailed below, tests a specified range of dimensions and returns the $\hat{d}$-bounded local dimension if it falls within the specified range.

Function: $\mathrm{LD}=$ LocalDimFinder $\left(f, z, \hat{d}, D_{0}, D_{1}\right)$

Inputs:

Complex analytic system $f: \mathbb{C}^{N} \rightarrow \mathbb{C}^{m}$,

Point $z \in Z=\mathcal{V}_{\mathbb{C}}(f)$,

Depth bound (integer) $\hat{d} \geq 1$,

Lower and upper dimension bounds (integer) $0 \leq D_{0} \leq D_{1}$.

\section{Outputs:}

$$
\mathrm{LD}= \begin{cases}\operatorname{dim}_{z, \hat{d}} Z, & \text { if } \operatorname{dim}_{z, \hat{d}} Z \in\left[D_{0}, D_{1}\right] ; \\ D_{0}, & \text { if } \operatorname{dim}_{z, \hat{d}} Z<D_{0} ; \\ \text { "not found", } & \text { if } \operatorname{dim}_{z, \hat{d}} Z>D_{1} .\end{cases}
$$

\section{Begin}

Set $d=0$ and, for $k=D_{0}, \ldots, D_{1}$, set $c_{k, 0}=1$ and $s_{k}=$ Continue.

For $d=1, \ldots, \hat{d}$

For $k=D_{0}, \ldots, D_{1}$, if $s_{k}=$ Continue, do the following:

Form $\mathcal{M}_{d}(z)$ for $S(f, k)$ and set $c_{k, d}=\operatorname{corank} \mathcal{M}_{d}(z)$.

If $c_{k, d}=c_{k, d-1}$, set $s_{k}=$ Stabilized.

If $\left\{k \mid s_{k}=\right.$ Stabilized $\}=\emptyset$, return LD $=$ "not found".

Otherwise, return LD $=\min \left\{k \mid s_{k}=\right.$ Stabilized $\}$.

End 

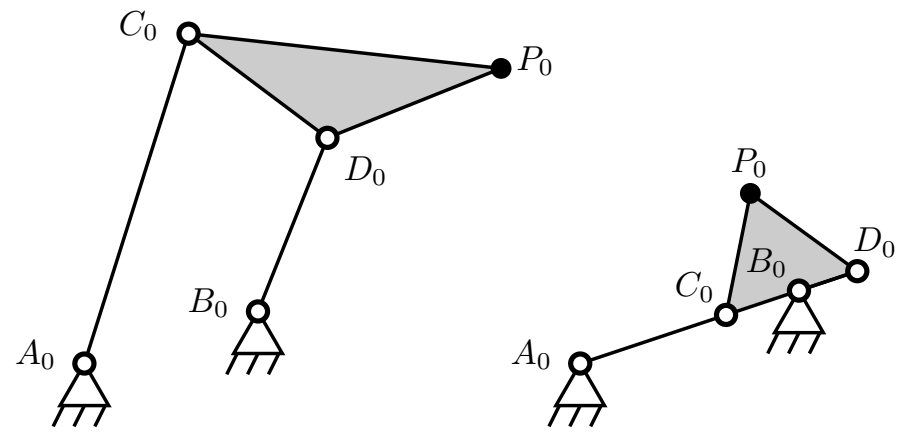

(a) $C_{0}=D_{0}$

(b)

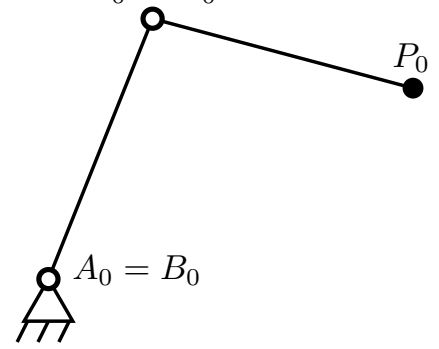

(c)

Figure 2: Planar four-bars: (a) general, (b) foldable, and (c) degenerate

The actual code also prints out the sequences $c_{k, d}$ and, as appropriate, gives messages equivalent to conclusions in (12) or (13). The code takes advantage of the multiprecision capabilities built into Bertini to accurately compute the required coranks. Since $z$ is given numerically, it will usually only be an approximate zero of $f$. It is the user's responsibility to make sure it is accurate enough to provide a meaningful answer.

In recent work, Zeng [27] has given a more efficient algorithm for computing the sequences $c_{d}(z)$. As of this writing, we have not yet revised LocalDimFinder to take advantage of this.

\section{Examples}

\subsection{Planar Four-bars}

It is well-known that a non-degenerate planar four-bar moves with one degree of freedom. We take this familiar example as an introductory illustration of the local dimension finding technique, testing a generic four-bar, a four-bar in a singular position, and a degenerate four-bar.

A pose of a planar four-bar with coupler point can be specified as the $(x, y)$ coordinates of five points, $A_{0}, B_{0}, C_{0}, D_{0}, P_{0}$, two of which, say $A_{0}$ and $B_{0}$ are 
the stationary ground pivots, while $C_{0}$ and $D_{0}$ are initial positions of the joints on the coupler link and $P_{0}$ is the initial position of the coupler point (see Fig. 2). After the linkage moves, the homologous points to $C_{0}, D_{0}, P_{0}$ are $C, D, P$ and the six coordinates of these are the variables in the assembly conditions. These conditions simply state that the links must have constant length, that is, $f(x)=$ 0 , where

$$
\begin{gathered}
f(x)=\left\{\begin{array}{l}
f_{1}=\left|C-A_{0}\right|^{2}-\left|C_{0}-A_{0}\right|^{2} \\
f_{2}=\left|D-B_{0}\right|^{2}-\left|D_{0}-B_{0}\right|^{2} \\
f_{3}=|C-D|^{2}-\left|C_{0}-D_{0}\right|^{2} \\
f_{4}=|P-C|^{2}-\left|P_{0}-C_{0}\right|^{2} \\
f_{5}=|P-D|^{2}-\left|P_{0}-D_{0}\right|^{2}
\end{array}\right. \\
x=(C, D, P)=\left(c_{x}, c_{y}, d_{x}, d_{y}, p_{x}, p_{y}\right) .
\end{gathered}
$$

The "point of construction," as we shall call $z=\left(C_{0}, D_{0}, P_{0}\right)$, is on $\mathcal{V}_{\mathbb{C}}(f)$, so we may check the dimension at that point. We have $n=5$ functions and $N=6$ variables, so by Eq. 3 ,

$$
N-n=1 \leq \operatorname{dim}_{z=\left(C_{0}, D_{0}, P_{0}\right)} \mathcal{V}_{\mathbb{C}}(f) .
$$

We will test the local dimension for three cases:

1. general;

2. foldable, i.e., $\left(A_{0}, B_{0}, C_{0}, D_{0}\right)$ collinear; and

3. degenerate with $A_{0}=B_{0}$ and $C_{0}=D_{0}$.

In terms of the discussion of $\S 7$, the parameter space for planar four-bars in the foregoing formulation is $\left(A_{0}, B_{0}, C_{0}, D_{0}, P_{0}\right) \in \mathbb{C}^{10}$. For each of the three families (general, foldable, degenerate), we can generate a random point satisfying the defining conditions. In the generic and degenerate cases, it is easy to see that the point of construction is a general point on the motion set of the linkage, while in the foldable case, the point of construction is a special point of the motion where the linkage is actually folded.

Calling LocalDimFinder with dimension range $[1,3]$ and depth bound $\hat{d}=5$ gives the results shown in Table 2. Since the dimension must be at least one, we have a conclusive result that the generic and foldable cases have dimension one, that is, the four bar has 1DOF. The foldable four-bar gives multiplicity two, which reflects the fact that the test point is at a self-crossing of the motion curve. The degenerate four-bar, which is in fact equivalent to a $2 \mathrm{DOF} R \mathrm{R}$ serial chain, is determined to have either a solution set of dimension 2 and multiplicity 1 or else it is dimension 1 with multiplicity 6 or greater. To use the local dimension test to definitively conclude that the true dimension is 2 , one would need an independent bound on the multiplicity and, if that is larger than 6 , one would need to compute the sequence $c_{1, d}$ farther. The a priori bound that the multiplicity cannot exceed the total degree of $f(x)\left(2^{5}=32\right)$ is too high to be practical. 


\begin{tabular}{|l|c|l|c|}
\hline case & $k$ & $c_{k, d}$ & stabilized? \\
\hline \multirow{4}{*}{ general } & 1 & 1,1 & yes \\
& 2 & 1,1 & yes \\
& 3 & 1,1 & yes \\
\hline \multirow{3}{*}{ foldable } & 1 & $1,2,2$ & yes \\
& 2 & 1,1 & yes \\
& 3 & 1,1 & yes \\
\hline \multirow{3}{*}{ degenerate } & 1 & $1,2,3,4,5,6$ & no \\
& 2 & 1,1 & yes \\
& 3 & 1,1 & yes \\
\hline
\end{tabular}

Table 2: Local dimension test results for the four-bar problems.

In all three cases, we are testing a generic four-bar from the indicated families, so we may conclude that the dimensions computed are the generic degrees of freedom of linkages in the respective families. In the foldable case, we see that $c_{1, d}$ has depth greater than zero, indicating that the test pose (the folded pose) is either singular or else the whole motion curve is nonreduced. We cannot tell the difference from the local test at this special point of construction. If we were to test a generic point of the motion curve, we would find that it is nonsingular, hence the motion curve is reduced and therefore the folded position is singular. This underscores that although the local dimension test at a singular point on a motion set gives a definitive answer for the degrees of freedom of the motion, the point is still special and hence may have a higher multiplicity than general points of the motion. Though it does not happen here, one must also keep in mind that it is possible for two or more motion components to meet at a singular point, and if so, the local dimension test will return the highest dimension among these. One does not get any signal whether or not lower dimensional components also exist.

\subsection{Foldable Griffis-Duffy 6SPU Platform}

A foldable Griffis-Duffy 6SPU platform is a type of Stewart-Gough platform that consists of two congruent equilateral triangles with legs of length equal to the altitude of the triangles that connect vertex to midpoint and midpoint to vertex sequentially around the triangles. This platform was studied in [17], where it was found to have a $1 \mathrm{DOF}$ motion that has seven real components. In the formulation of that paper, based on Study (soma) coordinates, these components are 3 double lines, 3 quadrics, and one quartic. The folded pose of the mechanism, in which the two triangles coincide while the legs lie along their common altitudes, is very singular, as it lies at the intersection of all three double lines and a self-crossing of the quartic. Thus, we expect the local dimension of the folded configuration to be one and its multiplicity to be 8 . For this mechanism, it happens that $\operatorname{rank} f=6$ and $N=7$, so we know the dimension must be at least 1 (see Eq. 3). Using LocalDimFinder with $k \in[1,3]$ 
and $\hat{d}=5$, we obtain the following three stabilized sequences:

$$
c_{1, d}=1,4,7,8,8 \quad c_{2, d}=1,3,3 \quad c_{3, d}=1,2,2 .
$$

The results of this computation match the expected dimension, 1 , and multiplicity, 8.

\subsection{The SNU 3UPU Parallel Robot}

A model of a parallel-link robot built at Seoul National University circa 2001 by F. Park and colleagues displayed an unexpected mobility. The device is a 3 -UPU mechanism, that is, it consists of two triangles with vertices connected by prismatically extending legs with universal joints at each end. The specific model had equilateral triangles of unequal size. In the "home" position, the leg lengths are all equal and the triangles are in parallel planes, one centered above the other. (That is, the line through their centers is perpendicular to the planes.) When the leg lengths are locked in this position, the mechanism was expected to be rigid, using the Grübler-Kutzbach formula, but the physical model allowed considerable motion. For studying cases with the leg lengths locked, we may consider the robot as a 3 -UU device.

Several analyses have been done that explain this behavior, with perhaps the most complete being one by Walter, Husty, and Pfurner [24]; our analysis is based on their equations (3.2-3.8). We applied LocalDimFinder to the home pose of the robot and found that

$$
c_{0, d}=1,3,4,4 .
$$

This reconfirms the statement made in [24] that the home pose is, in theory, an isolated solution point of multiplicity 4. (Additionally, we see that it has depth 2.) The high multiplicity allows the real device to move in the presence of joint tolerances and link elasticity. As this computation was done for a random size of the triangles and the leg length, we expect this behavior to persist generically. Of course, there may be exceptions that meet extra conditions whose dimensionality increases.

In fact, as discussed in [24], there do exist examples of the 3-UPU mechanism that have positive-dimensional solution components. One case, given in Eq. 5.2 of [24], is

$$
\left\{h_{1}=2 h_{2}, d_{1}=3 h_{2}, d_{2}=d_{3}\right\},
$$

where $h_{1}$ and $h_{2}$ are the circumradii of the base and upper triangles, resp., and $d_{1}, d_{2}, d_{3}$ are the three leg lengths. We consider a special case of this, namely

$$
\left\{h_{1}=2, h_{2}=1, d_{1}=d_{2}=d_{3}=3\right\} .
$$

For this case, the home position has the two triangles separated by distance $\sqrt{2}$. This point turns out to have sequence $c_{0, d}$ exactly the same as the general case above, that is, the home position is zero-dimensional with multiplicity 4 , 


\begin{tabular}{|c|c|c|}
\hline$k$ & $c_{k, d}$ & stabilized? \\
\hline 0 & $1,2,3,4,5,6$ & no \\
1 & 1,1 & yes \\
\hline
\end{tabular}

Table 3: Results for the 3-UU mechanism with parameters (18) at a point satisfying (19).

depth 2. However, for the same leg lengths, the mechanism can be assembled in a different mode, which using the notation of [24] is given as the line

$$
\left[x_{0}, x_{1}, x_{2}, x_{3}, y_{0}, y_{1}, y_{2}, y_{3}\right]=[0, \alpha, 0,-1,1,0,0,0], \quad \alpha \in \mathbb{R} .
$$

Testing a random point on this line, with the normalizing condition $y_{0}=1$, LocalDimFinder with $k \in[0,1]$ and $\hat{d}=5$ gives the results in Table 3 . We see that, as claimed in [24], the test point is on a 1DOF assembly mode. In fact, it has multiplicity 1 . (To be more accurate, we only know that either the mechanism has a one DOF motion or else it is rigid with multiplicity at least 6.)

\subsection{A Cubic-Centered 12-Bar}

The following example is included as an illustration of the difference between real dimension and complex dimension.

A collapsible cube, consisting of 12 scissors linkages each aligned with one edge of a cube, was presented in [25]. Locking the scissors, the mechanism becomes a 12-bar spherical linkage, with one link along each edge of a cube and with rotational joints at the vertices of the cube, all joint axes meeting at the center of the cube. Using the methodology espoused in [25], one may convert the links into triangles joined at their vertices. This comes down to a set of nine points, one for each vertex of the cube and its center as illustrated in Figure 3. Let us consider such a cube with side length 2 . To fix a ground link, we take the center point, $(0,0,0)$, and two adjacent vertices, say $P_{7}=(-1,1,-1)$ and $P_{8}=(-1,-1,-1)$, to be fixed. The remaining six vertices are free to move subject to maintaining their initial relative distances, that is, subject to:

$$
\begin{gathered}
f_{i j}=\left|P_{i}-P_{j}\right|^{2}-4, \\
\{i, j\} \in\{(1,2),(3,4),(5,6),(1,5),(2,6), \\
\quad(3,7),(4,8),(1,3),(2,4),(5,7),(6,8)\} ; \\
g_{i}=\left|P_{i}\right|^{2}-3, \quad i \in\{1,2,3,4,5,6\} .
\end{gathered}
$$

This is a total of $m=17$ equations in the 18 coordinates of points $P_{1}, \ldots, P_{6}$. Accordingly, we know that the dimension of the solution set is at least one dimensional. Indeed, if we test the assembly in which all of the points are at the nominal vertices of the cube, i.e.,

$$
\begin{aligned}
\left(P_{1}, \ldots, P_{6}\right)=((1,1,1),(1,-1,1),(1,1,-1), & (1,-1,-1),(-1,1,1),(-1,-1,1)),
\end{aligned}
$$




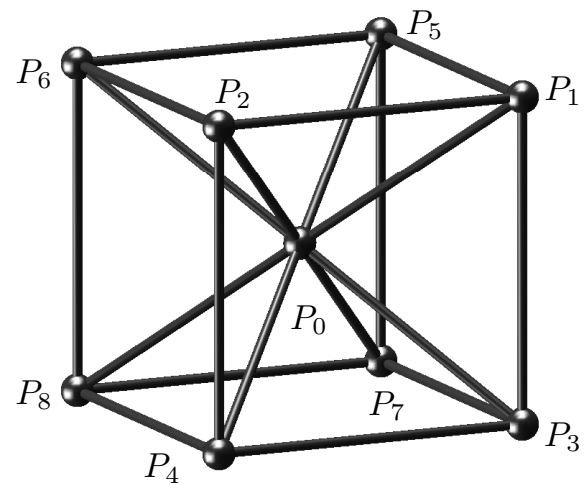

Figure 3: Cubic-Centered 12-Bar

LocalDimFinder gives the sequence $c_{1, d}=1,2,2$ indicating that the local dimension is 1 with multiplicity 2 and depth 1 . Since the multiplicity is greater than one, there is the possibility that the real dimension is less than the complex dimension. Indeed, this is true for the case at hand: the complex curve crosses itself in the home position, which turns out to be an isolated point over the reals.

If the mechanism is modified slightly by moving the central point off of the origin and adjusting the distances to it accordingly, then the mechanism does have a $1 \mathrm{DOF}$ real motion. The only change to the above equations is to replace $g_{i}$ with $\hat{g}_{i}$ as

$$
\hat{g}_{i}=\left|P_{i}-O\right|^{2}-\left|P_{i 0}-O\right|^{2}, \quad i=1, \ldots, 6,
$$

where $O$ is the central point and $P_{i 0}$ is the initial position of point $P_{i}$. As $O$ is moved towards the origin, one finds that the real $1 \mathrm{DOF}$ motion shrinks to a smaller and smaller loop approaching a single point in the limit. At the origin, an actual mechanism will retain a vestige of this motion as a wobbliness, an infinitesimal degree of freedom of multiplicity 2 .

It is interesting to note that the exact cubic-centered mechanism can be assembled in another mode where its real dimension and its complex dimension are both one. A point on this assembly curve is

$$
\begin{aligned}
\left(P_{1}, \ldots, P_{6}\right)=((-1,1,-1),(1,1,-1) & (1,1,-1), \\
& (1,-1,-1),(-1,-1,-1),(1,-1,-1)) .
\end{aligned}
$$

In this configuration, $P_{3}$ and $P_{4}$ are at their original positions, but the points originally on the upper face of the cube have been moved down to the lower face, with $P_{1}=P_{7}, P_{2}=P_{3}, P_{5}=P_{8}$, and $P_{6}=P_{4}$. The mechanism becomes an equilateral spherical four bar. 


\section{Conclusions}

We have given a methodology to determine the degrees of freedom of a mechanism for the assembly mode of a given configuration. The method is a generalization of testing the rank of the Jacobian matrix at the given configuration; specifically it works by testing the rank of certain arrangements of higher partial derivatives into matrices known as Macaulay matrices. We emphasize that the kinematicians' notion of degrees of freedom is precisely the same as the mathematicians' concept of dimensionality. When a mechanism has several assembly modes with different degrees of freedom, the applicable mathematical terminology is local dimension. The local dimension test described here is based on prior work [1] using multiplicity ideas from [3, 6]. Taken alone, the rank tests cannot distinguish between a finite degree of freedom and a high-multiplicity infinitesimal degree of freedom for which the Macaulay corank sequence stabilizes at a depth greater than the prespecified depth bound. However, we also describe several other inequalities that place limits on the possible dimensionality and these often provide an exact answer for the degrees of freedom. At the same time, when infinitesimal degrees of freedom are present, the Macaulay matrices provide multiplicity information that relates to the magnitude of motion one may expect in these directions.

The local dimension test treats the motion variables as complex numbers. Usually, the complex and real dimensionality of an assembly configuration are equal, but if the multiplicity is greater than one, the real dimensionality can be smaller. We give one example, the cubic-centered spherical 12-bar, where this occurs. The further elucidation of such cases to determine the local dimension in the reals will be the subject of future work.

The size of the Macaulay matrices, $\mathcal{M}_{d}$, grows quickly with depth, $d$, rapidly leading to unmanageable computations. Presently, our code is limited in this regard, but the closedness subspace method of [27] offers help for proceeding to greater depths.

\section{References}

[1] D.J. Bates, J.D. Hauenstein, C. Peterson, and A.J. Sommese. A numerical local dimension test for points on the solution set of a system of polynomial equations. SIAM Journal on Numerical Analysis, 47:3608-3623, 2009.

[2] D.J. Bates, J.D. Hauenstein, A.J. Sommese, and C.W. Wampler. Bertini: Software for numerical algebraic geometry. Available at http://www.nd.edu/ sommese/bertini.

[3] D.J. Bates, C. Peterson, and A.J. Sommese. A numerical-symbolic algorithm for computing the multiplicity of a component of an algebraic set. Journal of Complexity, 22:475-489, 2006.

[4] G.T. Bennett. A new mechanism. Engineering, 76:777-778, 1903. 
[5] D. Cox, J. Little, and D. O'Shea. Ideals, Varieties, and Algorithms. Springer-Verlag, New York, 1992.

[6] B. Dayton and Z. Zeng. Computing the multiplicity structure in solving polynomial systems. In Proc. of ISSAC 2005, pages 116-123, New York, 2005. ACM.

[7] E. Delassus. Les chaînes articulées fermées at déformables à quatre membres. Bulletin des Sciences Mathématiques et Astonomiques, 46:283-304, 1922.

[8] F. Geiss and F.-O. Schreyer. A family of exceptional Stewart-Gough mechanisms of genus 7. In D.J. Bates, G.-M. Besana, S. Di Rocco, and C.W. Wampler, editors, Interactions of Classical and Numerical Algebraic Geometry, volume Contemporary Mathematics 496, pages 221-234. Amer. Math. Soc., 2009.

[9] G. Gogu. Mobility of mechanisms: a critical review. Mechanism and Machine Theory, 40(9):1068-1097, 2005.

[10] M. Grübler. Getriebelehre: eine Theorie des Zwanglaufes und der ebenen Mechanismen. Springer, Berlin, 1917.

[11] J. Hauenstein, A.J. Sommese, and C.W. Wampler. Regeneration homotopies for solving systems of polynomials. Math. Comp., 80:345-377, 2011.

[12] J.M. Hervé. Analyse structurelle des mecanismes par groupe des deplacements. Mechanism and Machine Theory, 13:437-450, 1978.

[13] M.L. Husty, M. Pfurner, H.-P. Schröcker, and K. Brunnthaler. Algebraic methods in mechanism analysis and synthesis. Robotica, 25:661-675, 2007.

[14] M.L. Husty and H.-P. Schröcker. A proposal for a new definition of the degree of freedom of a mechanism. In A. Kecskeméthy, editor, Proc. Conf. on Interdisciplinary Applications of Kinematics (IAK2008), Lima, Peru, Jan. 9-11, 2008, 2008.

[15] A. Karger. Architecture singular planar parallel manipulators. Mechanism and Machine Theory, 38(11):1149-1164, Nov. 2003.

[16] K. Kutzbach. Mechanische leitungsverzweigung, ihre gesetze und anwendungen. Maschinenbau, der Betrieb, 8:710-716, 1929.

[17] Y. Lu, D. Bates, A.J. Sommese, and C.W. Wampler. Finding all real points of a complex curve. In Proceedings of the Midwest Algebra, Geometry and Its Interactions Conference, volume Contemporary Mathematics 448, pages 183-205. AMS, 2007.

[18] F.S. Macaulay. The Algebraic Theory of Modular Systems. Cambridge Univ. Press, 1916. 
[19] A. Müller. Generic mobility of rigid body mechanisms - on the existence of overconstrained mechanisms. In Proc. DETC/Mechanisms $\&$ Robotics Conf., Sept. 4-7, Las Vegas, NV (CDROM). ASME, 2007. Paper DETC2007-34621.

[20] J. M. Rico, J. Gallardo, and B. Ravani. Lie algebra and the mobility of kinematic chains. Journal of Robotics Systems, 20:477-499, 2003.

[21] J. M. Rico and B. Ravani. On calculating the degrees of freedom or mobility of overconstrained linkages: single-loop exceptional linkages. J. Mech. Design, 129(3):301-311, 2007.

[22] A.J. Sommese, J. Verschelde, and C.W. Wampler. Advances in polynomial continuation for solving problems in kinematics. Journal of Mechanical Design, 126(2):262-268, 2004.

[23] A.J. Sommese and C.W. Wampler. The Numerical Solution of Systems of Polynomials Arising in Engineering and Science. World Scientific, Singapore, 2005.

[24] D.R. Walter, M.L. Husty, and M. Pfurner. A complete kinematic analysis of the SNU 3-UPU parallel robot. In D.J. Bates, G.-M. Besana, S. Di Rocco, and C.W. Wampler, editors, Interactions of Classical and Numerical Algebraic Geometry, volume Contemporary Mathematics 496, pages 331346. Amer. Math. Soc., 2009.

[25] C. Wampler, B. Larson, and A. Erdman. A new mobility formula for spatial mechanisms. In Proc. DETC/Mechanisms 83 Robotics Conf., Sept. 4-7, Las Vegas, NV (CDROM). ASME, 2007. Paper DETC2007-35574.

[26] K. Wohlhart. Kinematotropic linkages. In J. Lenarcic and V. ParentiCastelli, editors, Recent Advances in Robot Kinematics, pages 359-368. Kluwer Academic Publishers, 1996.

[27] Z. Zeng. The closedness subspace method for computing the multiplicity structures of a polynomial system. In D.J. Bates, G.-M. Besana, S. Di Rocco, and C.W. Wampler, editors, Interactions of Classical and Numerical Algebraic Geometry, volume Contemporary Mathematics 496, pages 347362. Amer. Math. Soc., 2009.

[28] J.-S. Zhao, Z.-J. Feng, and J.-X. Dong. Computation of the configuration degree of freedom of a spatial parallel mechanism by using reciprocal screw theory. Mechanism and Machine Theory, 41(12):1486-1504, 122006. 